\title{
Animal-in-the-loop System with Multimodal Virtual Reality to Elicit Natural Olfactory Localization Behavior
}

\author{
Shunsuke Shigaki, ${ }^{*}$ Natsuki Minakawa, ${ }^{1}$ Mayu Yamada, ${ }^{1}$ \\ Hirono Ohashi, ${ }^{1}$ Daisuke Kurabayashi, ${ }^{2}$ and Koh Hosoda ${ }^{1}$ \\ ${ }^{1}$ Graduate School of Engineering Science, Osaka University, \\ 1-2 Machikaneyama-cho, Toyonaka, Osaka 560-0043, Japan \\ ${ }^{2}$ Department of Systems and Control Engineering, Tokyo Institute of Technology, \\ 2-12-1 Ookayama, Meguro-ku, Tokyo 152-8552, Japan
}

(Received August 31, 2021; accepted December 8, 2021)

Keywords: animal-in-the-loop, multimodal virtual reality, olfactory localization, male silkworm moth

Herein, we proposed an "animal-in-the-loop" (AIL) system by introducing a robot and a virtual reality (VR) technology in a conventional insect behavior experiment. The setup provided sensory inputs to an insect, which mimic its natural environment and simultaneously measured the behavioral output. The proposed AIL system consisted of a multimodal VR device and a ground-running robot, both of which were connected wirelessly. The insect behavior was measured using a multimodal VR device, and the behavioral changes were transmitted to the robot as control inputs. Specifically, the multimodal VR device was equipped with three types of sensory stimulators, odor, wind, and vision, and each stimulator was controlled by the value of the corresponding sensor on the robot. The surrounding environment was observed using multiple sensors mounted on the robot, and the information was transmitted to the VR device to provide sensory stimuli to the insect. This system allowed the insect on the VR device to remotely control the robot and perform localization virtually. The localization trajectories of the proposed AIL system were similar to those of the free-walking experiment, and the tendency of the change in the heading angle during localization was also similar. Therefore, we found that using the AIL system enabled us to measure behavioral changes upon providing sensory stimuli to insects. These VR stimuli were similar to those encountered by the insect in free-walking experiments.

\section{Introduction}

Animals modulate their behavior according to sensory information acquired from the surrounding environment. By selecting suitable behaviors in response to the environment, also called adaptive behavior, animals can survive and flourish. Adaptive behavior is an interesting phenomenon for not only biologists but also engineering researchers, and they have formulated methods to elucidate the information-processing mechanisms that may help reconstruct adaptive behavior that can be used to control a robot. ${ }^{(1,2)}$ In particular, researchers have actively investigated adaptive behavior in localization, ${ }^{(3,4)}$ which is a fundamental behavior in animals. *Corresponding author: shigaki@arl.sys.es.osaka-u.ac.jp https://doi.org/10.18494/SAM.2021.3609 
To perform localization, animals select an optimized route while acquiring environmental cues through sensory organs. Therefore, the elucidation of sensory-motor integration is well studied, and experiments have been conducted to analyze changes in behavior and neural activity when sensory inputs are provided under different conditions. ${ }^{(5)}$ Previous studies including Ref. 5 have revealed some of the sensory and motor integration, but many such biological experiments were conducted in a fixed environment with a constant amount and period of stimulation. Experiments conducted in a fixed environment are useful for identifying specific neural circuits or brain regions in animals. However, in the natural environment, sensory stimuli of the same intensity for a fixed duration are unlikely. Moreover, a conventional bio-inspired algorithm has been developed on the basis of data from biological experiments conducted in an environment with fixed stimulus conditions; therefore, a robot implemented with this algorithm works well under some environmental conditions but cannot cope with all environments. ${ }^{(6)}$ To overcome this problem, it is important to accurately determine the relationship between the sensory input and the behavioral output of animals moving in a natural environment. This shows that the behavior peculiar to a fixed environment may be elicited in a conventional biological experiment, and the real localization behavior is not measured accurately. Hence, virtual reality (VR) research to measure the behavior of insects has become active in recent years. ${ }^{(7-9)}$ Because a VR device can simultaneously and continuously provide odor, vision, sound, and wind stimuli to an insect, it can acquire the combined behavioral output of multiple sensory inputs. The VR device is commonly connected to a virtual environment (VE) created in a computer, and environmental changes in the VE are provided to the insect as sensory inputs. Moreover, changes in the insect behavior are transmitted to the VE agent so that the insect can move virtually. Therefore, VR devices have attracted attention as a useful tool for investigating adaptive behavior during localization. However, because the VE is an ideal environment, it is difficult to mathematically model all the phenomena that occur in the real environment; therefore, the knowledge gap between real and ideal environments needs to be addressed urgently.

We have thus proposed a novel experimental system called the "animal-in-the-loop" (AIL) system by introducing robot technology into conventional biological behavior experiments. ${ }^{(10)} \mathrm{A}$ conceptual diagram of the AIL system is shown in Fig. 1. The animal (insect) remotely manipulates a robot in real space. This system replaces the connection destination of the VR device with a robot that moves in the real environment instead of the ideal VE (Fig. 1). Specifically, the robot is equipped with a sensor group corresponding to the sensory organs of the insect, and the values measured by the sensors are provided to the insect as environmental information via each stimulator of the VR device. When environmental information is provided as sensory input, the insect behaves in response to stimuli; thus, by using the behavioral output of the insect as the speed input of the robot, the movement of the insect is reflected in the robot. This system allows the insect to virtually achieve a task in the real environment. We provided only odor stimulation to the insect and showed that localization could be achieved even if the insect manipulated a robot whose movement dynamics were completely different from those of the insect. However, because the localization trajectory was completely different from that of the free-walking experiment of the insect, it is necessary to present other sensory cues in addition to odor. 


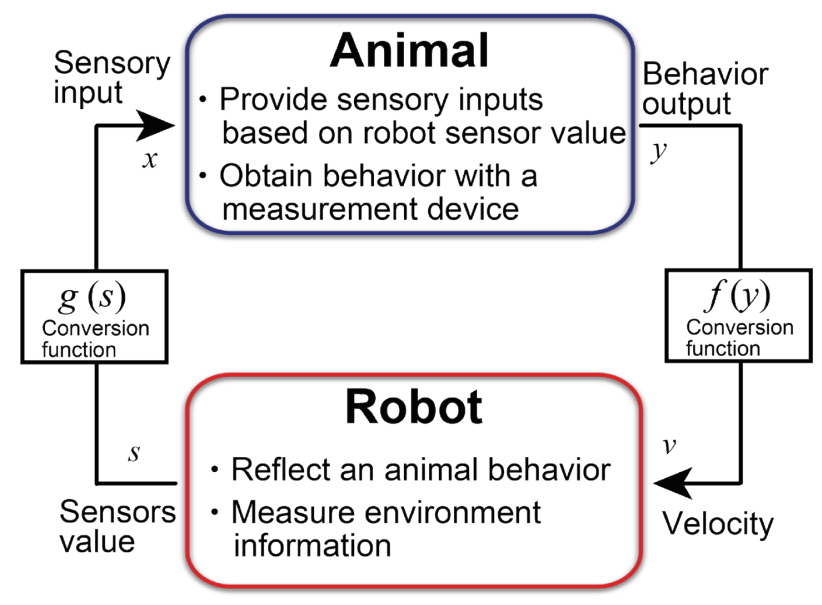

Fig. 1. (Color online) Conceptual diagram of an animal-in-the-loop system.

Therefore, in this study, we investigated whether it is possible to replicate the behavioral trajectory of an insect when it walks freely in the natural environment by connecting a VR device capable of providing multiple stimuli and a robot equipped with multiple sensors. If the trajectory is equivalent to that of a freely walking insect, it means that the sensory stimulus from the environment can be reconstructed, and the proposed system can be used as a tool for measuring the adaptive behavior of insects in the future. In this study, the trajectory of the freewalking experiment was used as a control group, and (1) the trajectory when the VR device was connected to the robot and (2) the trajectory when the VR device was connected to the VE were compared. We experimentally demonstrated that the sensory stimuli received from the real environment can be reproduced by connecting the multisensory VR and the robot. If this extended AIL system is used effectively, it can enable us to obtain the input/output relationship of an insect in an open field, which could not be measured in a fixed-stimulus environment. In addition, we are able to reconstruct an adaptive controller for the insect using the experimental data. We believe that extended AILs can contribute to the effective combination of biological and engineering concepts.

\section{Problem Statement}

In this study, we aim to construct an experimental system to acquire the dataset of sensory input and behavioral output necessary for analyzing the adaptive behavior of an insect. To elucidate adaptive behavior, we need to acquire accurate information to validate the relationship between sensory input and behavioral output in a real environment. However, in a conventional free-walking experiment in a wind tunnel, it is difficult to measure when and how sensory stimuli are received by insects. Moreover, it is not possible to quantify the extent to which sensory information is reflected in insect behavior. Various behavioral experimental devices have been developed to acquire these input/output relationships. One such device is a tethered system, ${ }^{(11,12)}$ in which a part of the insect body is fixed; the other is a servosphere, ${ }^{(13,14)}$ in which 
visual feedback can compensate for the movement of a free-walking insect and measure its behavior without tethering and spatial limitations. The limitation of these experimental systems is the disparity between the stimulus intensity presented to the insect under controlled conditions and that in the real environment. To solve this problem, we attempted to connect a behavior measurement device to a VE built on a computer. ${ }^{(9)}$ However, it remains uncertain whether the odor diffusion stimulus mimics that of the natural environment. Therefore, in this study, we propose an extended AIL system that can provide sensory stimuli as if the insect moves in a real environment while recording the relationship between sensory input and behavioral output with high accuracy. The extended AIL system consists of the following two elements:

- a VR device that provides multisensory stimuli to an animal and measures its behavior, and

- a robot that moves in the real environment as an avatar of the animal.

In this study, we measured the female search behavior of an adult male silkworm moth, Bombyx mori (Lepidoptera: Bombycidae), as a model animal. The adult male silkworm moth has been reported to elicit search behavior in response to female sex pheromones (bombykol), and behavioral modulation occurs depending on the amount of visual and wind stimuli acquired. $(15,16)$ Therefore, the VR device is equipped with a sense of odor, vision, and a wind stimulator. In addition, the robot that becomes the avatar of the silkworm moth is equipped with odor sensors and wind sensors. The VR device and the robot are wirelessly connected, and the amount of movement of the silkworm moth and the multisensory information acquired by the robot are exchanged. Note that the diffusion characteristics of ethanol and bombykol are almost the same. ${ }^{(17)}$ By conducting a localization experiment, we verified that the silkworm moth followed the same trajectory as that during the free-walking experiment in the natural environment using the constructed extended AIL system. With the extended AIL system, the robot moved in the real environment instead of the silkworm moth, and the robot was equipped with an odor sensor that reacts with alcohol with high sensitivity; therefore, we used ethanol as an odor source. The experimental results obtained with the extended AIL system were compared with those of the free-walking experiment and those obtained with the AIL system connected to a VE. A quantitative comparison showed the effectiveness of the extended AIL system in generating similar search trajectories to those in the free-walking experiment. Furthermore, if the trajectory of the extended AIL system more closely matches that in the free-walking experiment than the localization trajectory when connected to the VE, we can conclude that multisensory information and environment dynamics that are difficult to express in a mathematical model can be appropriately reconstructed and provided by this method.

\section{Construction of Extended Animal-in-the-Loop System}

A conceptual diagram of the extended AIL system is presented in Fig. 2. The extended AIL system consists of a multimodal VR device and a robot that moves in real space as an avatar of a silkworm moth. The robot is equipped with multiple sensors, and the values measured by them are provided to the silkworm moth as multisensory information via each stimulator on the VR device. The silkworm moth changes its behavior upon receiving sensory stimuli, and the behavior output of the silkworm moth is input as the control amount of the robot. By exchanging 


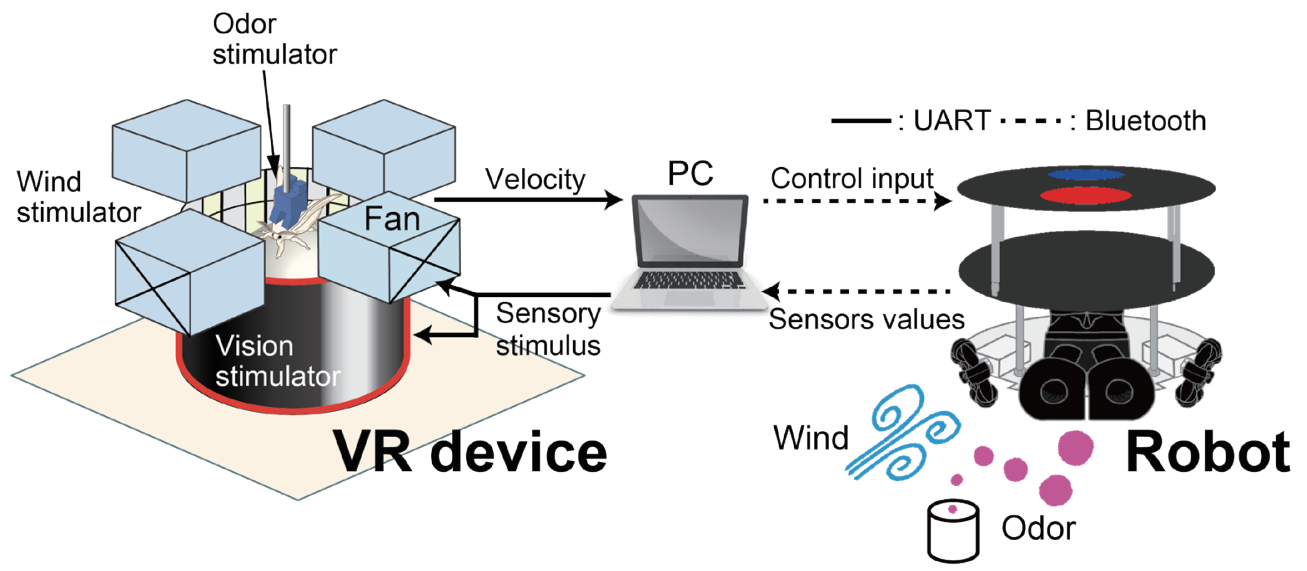

Fig. 2. (Color online) System configuration of the extended AIL. Communication between the VR device and the robot was performed using Bluetooth.

multisensory information and the amount of movement between the VR device and the robot, the relationship between the sensory input and the behavior output of the silkworm moth moving in the real environment is acquired. The communication delay between the multimodal VR device and the robot is about $30 \mathrm{~ms}$. A detailed explanation of the VR device and robot is provided in the following sections.

\subsection{Multimodal VR}

An outline of the multimodal VR device is shown in Fig. 3. We employed a tethered system that fixed a part of an insect body and measured its behavior ${ }^{(11)}$ because it is important to provide accurate sensory stimulation to the insect. In the tethered system, because the insect was fixed on the top of a passively rotating sphere, the movement of the legs was transmitted to the sphere, so that the amount of rotation of the sphere was measured as the amount of movement of the insect. We used a chloroprene rubber adhesive (G17, Konishi Co. Ltd., Osaka, Japan) to glue a tethered rod to the back of a silkworm moth. The passively rotating sphere was lifted by wind blowing from below the sphere, and the amount of sphere rotation was read by two optical sensors (MAMA6W, Sanwa Supply, Okayama, Japan).

Odor, vision, and wind stimulators were equipped around the insect in the multimodal VR. An odor stimulator was used, which consisted of an air compressor (NIP30L, Nihon Denko, Aichi, Japan), three gas washing bottles (absorbent cotton, activated carbon, and distilled water), a flow meter (KZ-7002-05A, AS ONE Corporation, Osaka, Japan), and a solenoid valve (VT307, SMC Corporation, Tokyo, Japan). The air exhausted from the air compressor passed through the absorbent cotton, activated carbon, and distilled water, was adjusted to a constant flow rate of $1.0 \mathrm{~L} / \mathrm{min}$ using a flow meter, and was finally exposed to the silkworm moth. The odor discharge port was integrated with the tethered rod, and the sex pheromone (bombykol) was provided to the upper part of the antennae of the male silkworm moth.

Because the silkworm moth has compound eyes and therefore shows a strong response to an optical flow, ${ }^{(15)}$ we employed an array of white LEDs of approximately $300 \mathrm{~cd} / \mathrm{m}^{2}$ (WS2812B, 


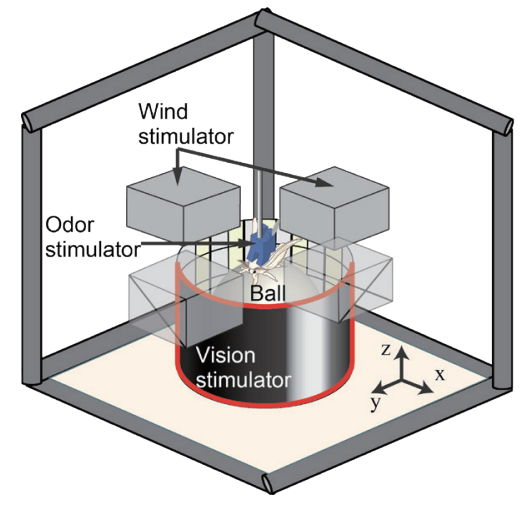

(a)

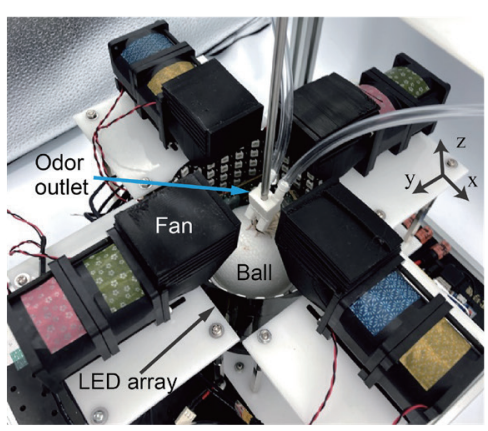

(b)

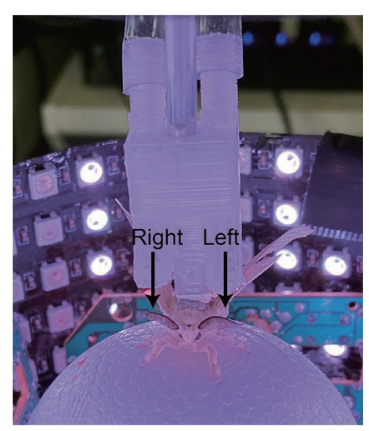

(c)

Fig. 3. (Color online) (a) Schematic diagram of multimodal VR device. (b) Arrangement of each sensory stimulator. (c) Enlarged view of the odor outlet, which can present stimuli independently to the left and right antennae.

WORLDSEMI Co., Ltd., Guang Dong, China) as a visual stimulator. Because the width of the optical flow pattern was $20 \mathrm{~mm}$ and the distance from the silkworm moth eye to the visual stimulator was $50 \mathrm{~mm}$, the spatial frequency was calculated as an absolute angle of about $21^{\circ}$ using the following equation:

$$
\theta=\tan ^{-1} \frac{l}{r}
$$

where $l$ and $r$ represent the pattern width and the distance to the stimulator, respectively. This LED array is controlled according to the angular velocity of the robot, and when the robot rotates counterclockwise, the direction of the optical flow is clockwise and vice versa. This gives the illusion that the silkworm moth is turning.

A wind stimulator generates an airflow based on the principle of a push-pull rectifier. ${ }^{(18)}$ The push-pull rectifier we used consists of two fans (PMD1204PQB1, SUNON, Takao, Taiwan) on the push and pull sides, and produces a wind of approximately $1.0 \mathrm{~m} / \mathrm{s}$. In previous research on measuring the response of the silkworm moth to wind speed, it was found that behavioral changes occur in response to winds of over $1.0 \mathrm{~m} / \mathrm{s} ;{ }^{(16)}$ therefore, we provided a wind speed of $1.0 \mathrm{~m} / \mathrm{s}$. The wind sensor on the robot outputs an analog voltage value for the wind speed. However, when the search field of the localization experiment is small, the sensor does not change its value significantly because of the low resolution of the wind sensor; thus, the wind speed of the environment may not be measured correctly. Therefore, in this experiment, we modulated the direction from which the wind arrived and fixed the wind speed. Two sets of push-pull rectifiers were installed on the $x$ and $y$ axes to provide the wind from the front, back, left, and right of the silkworm moth.

Each of these stimulators was controlled according to the values of the multiple sensors of the robot. The odor stimulus providing timing to the silkworm moth is the rise detection timing of the odor sensor. Moreover, the results of estimating the wind direction from the four wind 
sensors mounted on the robot are presented to the silkworm moth as wind stimuli. The visual stimulus determines the direction of the optical flow provided to the silkworm moth, which depends on the direction of rotation of the robot.

\subsection{Multimodal robot platform}

Figure 4 shows a ground-running robot that moves in the real environment as an avatar of a silkworm moth. The ground-running robot has a cylindrical shape with a diameter of $80 \mathrm{~mm}$ and consists of a measurement system and a drive system. It also has a microcomputer (ESP32, Espressif Systems, Shanghai, China) to control the measurement system and drive system. Communication with the multimodal VR is performed via Bluetooth (Fig. 2).

The role of the measurement system is to grasp the state of the environment around the robot using multiple sensors. The system is equipped with an odor sensor (MiCS-5524, SGX Sensortech Ltd., Neuchätel, Switzerland) and a wind sensor (Wind Sensor Rev. P, Modern Device, Rhode Island, USA). Because the odor is detected by the two antennae on the head of the silkworm moth, the robot is also equipped with two odor sensors corresponding to the antennae.

We used ethanol as the odor source because the sensor is highly sensitive to ethanol. Note that the diffusion characteristics of ethanol and bombykol are almost the same. ${ }^{(17)}$ The odor sensor used is capable of detecting odor frequencies up to $5 \mathrm{~Hz},{ }^{(19)}$ which is sufficient for the frequency of the odor emitted by a female silkworm moth (approximately $1.0 \mathrm{~Hz}$ ). ${ }^{(20)}$ Because the rise times of the electroantennogram (electric potential response of antennae) and the MiCS5524 odor sensor are the same, ${ }^{(21)}$ we detect the rise timing based on the values of the odor sensor and provide an odor stimulus to the silkworm moth at the time of the rise. When the left (right) odor sensor reacts, the odor stimulus is presented to the left (right) antenna of the silkworm moth. When both odor sensors respond, odor stimuli are presented to both antennae. In addition, the silkworm moth has a "fluttering effect" that draws in the odorant in its direction by flapping and eliminates the odor from behind;(22,23) therefore, we installed a small fan behind

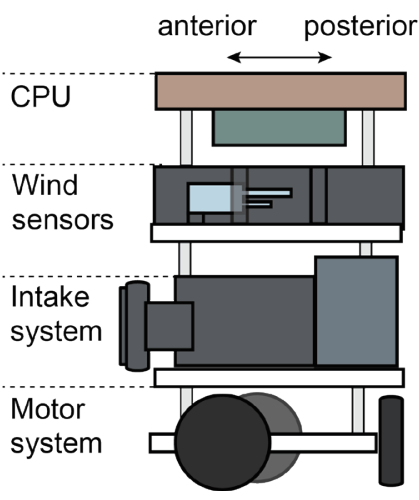

(a)

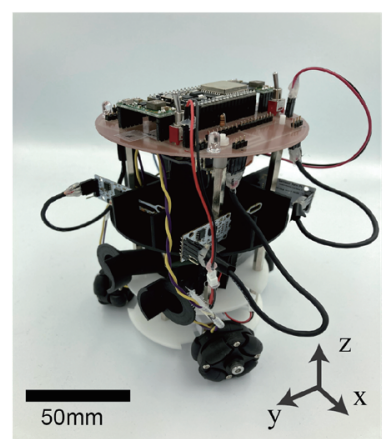

(b)

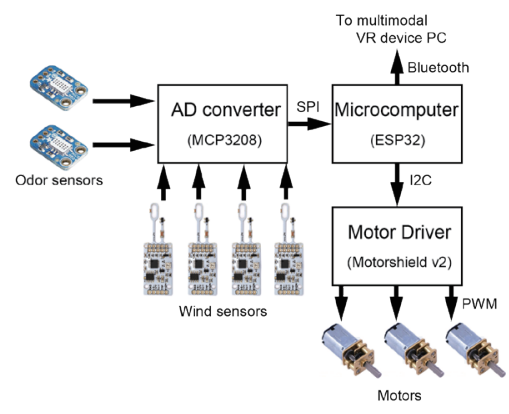

(c)

Fig. 4. (Color online) Schematic diagram of a ground-running robot. (a) Explanation of each level. (b) Appearance of the actual robot. (c) System configuration. 
the odor sensors to produce the same effect as shown in Fig. 5(a). The device that produces the artificial fluttering effect with the fan is called the intake system for convenience, and its function was evaluated experimentally. The ethanol source was placed to the front, back, left, and right of the intake system, and the detection rate was measured 10 times when ethanol was released. As shown in Fig. 5(b), the detection rate for odors from the front was high but it was zero for odors from the rear. We determined which antennae received the sex pheromone by controlling the values of the left and right odor sensors.

To detect the wind direction, we installed four wind sensors on the front, back, left, and right of the robot, ${ }^{(24)}$ as shown in Fig. 6(a). In addition, we designed partitions at both ends of the wind sensor element to improve its directivity. ${ }^{(25)}$ When the robot was exposed to wind from the four directions (front, back, left, and right), it was found that it was able to correctly detect the wind direction, as shown in Fig. 6(b). We also measured the values of the wind sensor when the robot underwent rotational motion (approximately $1 \mathrm{rad} / \mathrm{s}$ ) and moved directly toward the wind source (approximately $150 \mathrm{~mm} / \mathrm{s}$ ) [Figs. 6(c)-6(f)]. Under the no wind condition, we confirmed that the wind sensor was not affected by the rotational motion or by the robot moving directly forward [Figs. 6(c) and 6(e)]. Under the wind conditions, the values of the wind sensor facing upwind were highest, indicating that the wind direction could be estimated [Figs. 6(d) and 6(f)]. However, because the wind sensor values do not dramatically increase when the distance to the wind source decreases owing to the limited detection resolution of the sensor, the wind speed is not fed back to the insect, and only the wind direction is focused on. On the basis of the results, we provided wind direction stimulation to the silkworm moth depending on the values from the four wind sensors.

Furthermore, the optical flow direction was determined according to the amount of rotational control input to the robot because the visual stimulus presented the direction in which the robot was turning. Because no angular velocity is generated during ideal straight motion, the optical flow was set to stop. However, ideal straight motion without angular velocity was not observed during the localization experiment (see Sect. 4).

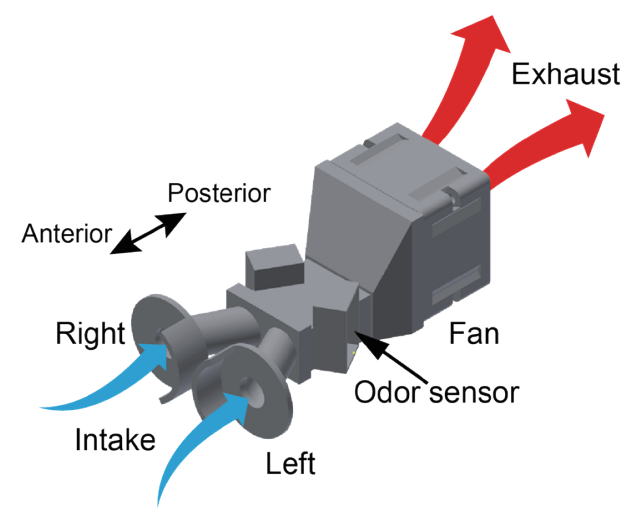

(a)

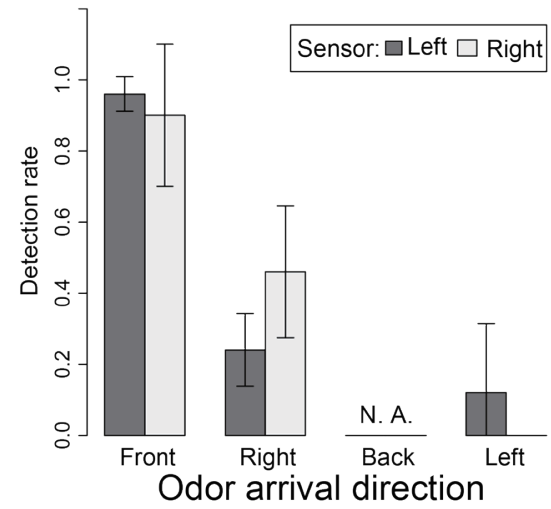

(b)

Fig. 5. (Color online) Schematic diagram of the intake system and the results of the odor directivity experiment. The intake system drew an odor from the front and exhausted it to the rear. The highest detection rate was in the front direction. 


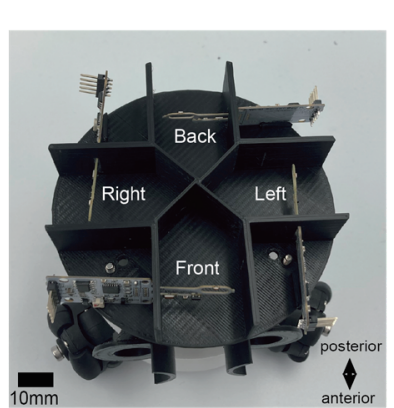

(a)

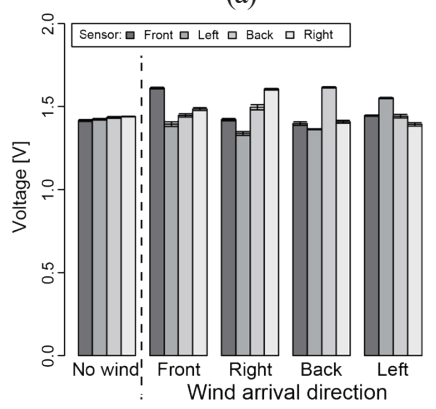

(b)

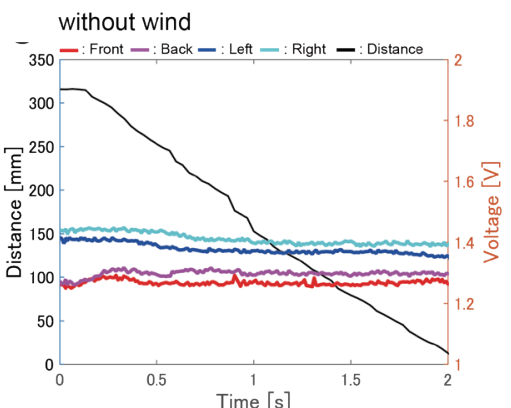

(c)

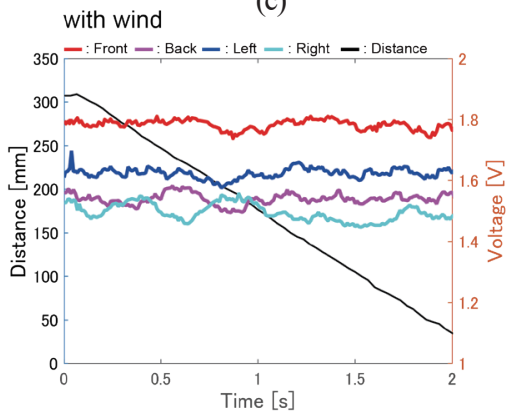

(d)

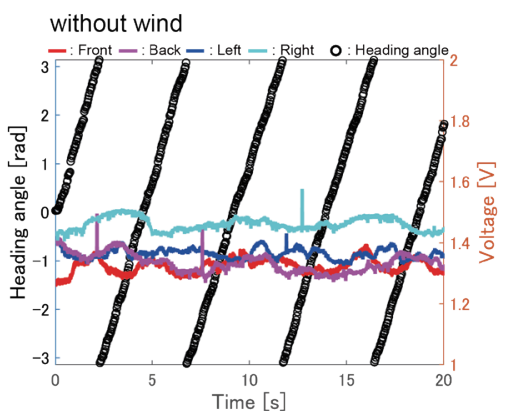

(e)

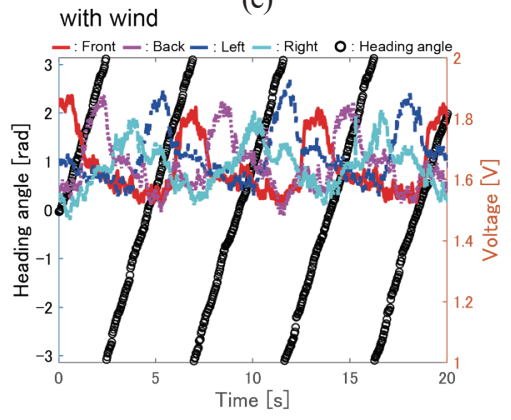

(f)

Fig. 6. (Color online) Wind sensor arrangement and wind direction detection experiment results. (a) Arrangement of wind sensors. (b) Response of the wind sensors when the wind is presented from each direction. The wind sensor values are higher in the direction of the wind than under the no wind condition. (c, d): Changes in the response of the wind sensors when the robot is moving directly toward the wind source. Under the no wind condition, the value of the front wind sensor (red line) is not high, but it is high under the wind condition. (e, f): Changes in the response of the wind sensors during the rotational motion of the robot at a distance of $300 \mathrm{~mm}$ from the wind source. Under the no wind condition, there is no change in the output values of the wind sensors, but when there is wind, the sensor values fluctuate periodically.

The silkworm moth may move in a direction orthogonal to the direction of travel because it is a six-legged insect. Therefore, we employed three omni-wheels $(38 \mathrm{~mm}$ plastic omni-wheel, Nexus robot, Hong Kong, China) for an omnidirectional drive system. The three omni-wheels were arranged at $2 \pi / 3$ rad intervals, as shown in Figs. 4(a) and 4(b), and the kinematic model of the three omni-wheels is as follows:

$$
\left[\begin{array}{l}
\Omega_{1} \\
\Omega_{2} \\
\Omega_{3}
\end{array}\right]=\left[\begin{array}{ccc}
1 & 0 & R \\
-1 / 2 & -\sqrt{3} / 2 & R \\
-1 / 2 & -\sqrt{3} / 2 & R
\end{array}\right]\left[\begin{array}{l}
V_{x} \\
V_{y} \\
V_{\theta}
\end{array}\right],
$$

where $\Omega_{1}-\Omega_{3}$ represent the amount of rotation of each omni-wheel and $R$ is the radius of the robot. Moreover, $V_{x}, V_{y}$, and $V_{\theta}$ represent the velocities of the silkworm moth in the directions indicated by the subscripts. According to Eq. (2), when the velocities of the silkworm moth are input, the amount of rotation of each omni-wheel is determined to reflect the velocities of the silkworm moth. Because the geared motor (HPCB 6V 298:1, Pololu Corporation, Nevada, USA) 
that rotates the omni-wheels is controlled by pulse width modulation (PWM), we calibrated the relationship between the actual amount of omni-wheel rotation and the amount of PWM in advance. In this study, the velocities of the silkworm moth and robot were set to be the same.

\section{Olfactory Localization Experiments}

\subsection{Experimental conditions}

We conducted three types of olfactory localization experiments to determine whether a realworld environment can be reconstructed by an extended AIL system: (1) a free-walking experiment, (2) an experiment with the AIL system connected to a VE, and (3) an experiment with the AIL system connected to a ground-running robot. When the results of experiments (1) and (2) were used as the control, the result of experiment (3) was used in the proposed method. Furthermore, in experiments using the AIL system, we set two types of conditions: (i) providing all sensory stimuli of odor, wind, and vision and (ii) providing only odor. This made it possible to investigate whether the characteristics of the elicited localized behavior were caused by the provision of multiple stimuli or by the connection with the robot. All localization experiments were carried out in an area of approximately $1 \mathrm{~m}$ square, the odor source was set to the origin $(x, y)=(0,0)$, and the initial position for localization was set to $300 \mathrm{~mm}$ leeward from the odor source, $(x, y)=(300,0)$. A fan installed behind the odor source generated wind of approximately $0.6 \mathrm{~m} / \mathrm{s}$ at the odor source. This wind speed was almost the same as that of the past behavioral experiments on silkworm moths. ${ }^{(26)}$ We selected the frequency of odor discharge to be $1 \mathrm{~Hz}$ (duration: $0.2 \mathrm{~s}$, interval: $0.8 \mathrm{~s}$ ) because a female silkworm moth emits sex pheromones at a frequency of approximately $1 \mathrm{~Hz} \cdot{ }^{(20)}$ A successful localization was identified as the case when an agent (robot/silkworm moth) approached the odor source. If the agent could not find the odor source within $300 \mathrm{~s}$ from the start of localization, we defined the localization as a failure. The behavior of the agent moving in a real environment was measured using a camera (BSW200MBK, BUFFALO, Aichi, Japan) installed above the experimental field. We acquired the trajectory of the agent by applying DeepLabCut ${ }^{(27)}$ to the recorded video and analyzed the trajectory. Details of each localization experiment are described below.

(1) Free-walking experiment

In this experiment, $1000 \mathrm{ng}$ of bombykol was placed at the odor source, and an adult male silkworm moth began searching from the initial position. An experiment was conducted using 10 silkworm moths.

(2) Experiment with AIL system connected to VE

The video-based odor model proposed by Yanagawa et al. ${ }^{(28)}$ was employed for odor diffusion in a VE. The video-based odor model is a method of reproducing the behavior of actual fine particles (particle diameter: $<10 \mu \mathrm{m}$ ) in a VE through image processing (Fig. 7). The behavior of fine particles was captured using the same technique as that used for particle image velocimetry (PIV). ${ }^{(29)}$ A laser sheet was placed in the actual experimental field, and fine particles were scattered on the laser sheet. We captured their behavior with a high-sensitivity camera. The advantage of this method is that the simulated odor diffusion is close to that in 


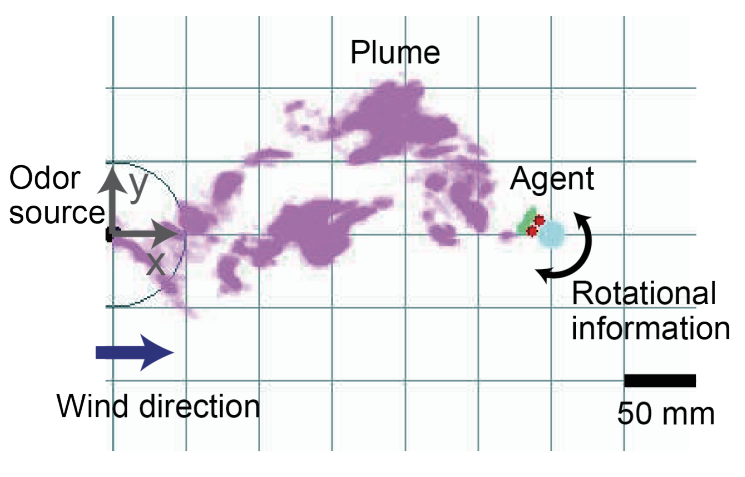

(a)

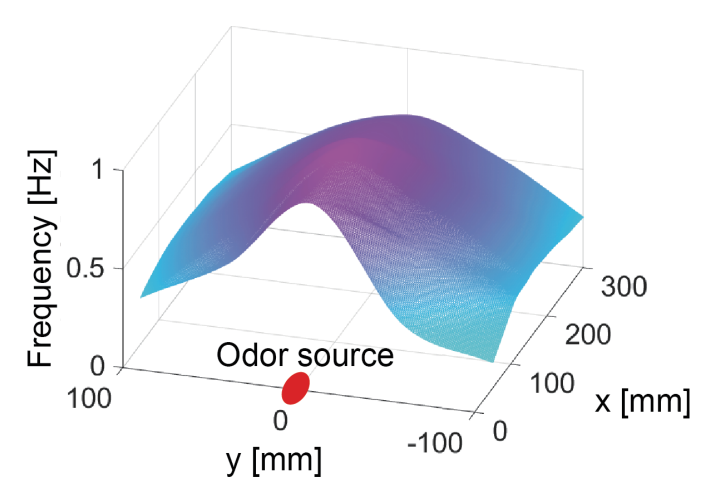

(b)

Fig. 7. (Color online) (a) Outline of VE. (b) Frequency characteristics of odor field.

the real environment as the actual behavior of fine particles was used as a model for odor diffusion. The speed of the wind used to diffuse the odor and the odor release frequency were set to $0.6 \mathrm{~m} / \mathrm{s}$ and $1 \mathrm{~Hz}$, respectively, which are the same as in other experiments. An agent that reflects the movement of the silkworm moth was installed in the virtual odor field, and a localization experiment was conducted. The agent on the virtual odor field starts localization at a distance of $300 \mathrm{~mm}$ from the odor source. The VR device used was the same as that in the previous section. In this experiment, we employed 10 silkworm moths. In the experiment with the connection to the VE, we provided multiple sensory stimuli of odor, wind, and vision to the silkworm moth. The wind direction to be presented to the silkworm moth was determined according to the heading angle of the agent because the wind flows in the $+x$ axis direction in the VE as shown in Fig. 7. The resolution of the wind direction corresponds to the four directions of front, back, left, and right. The optical flow provided as a visual stimulus was determined by the direction of rotation of the agent. These settings are the same as those in the experiment with the connection to the robot.

(3) Experiment with AIL system connected to ground-running robot

In this experiment, the proposed AIL system was used, which is a system in which the VR device is connected to a robot as discussed in the previous section. In this experiment, ethanol $(99.5 \%)$ was installed as the odor source, and the robot started localization at a distance of $300 \mathrm{~mm}$. In this experiment, 10 silkworm moths were used.

The silkworm moths used in the experiment were purchased from Ehine Sansyu Co., Japan. Adult male moths were cooled at $16{ }^{\circ} \mathrm{C}$ after eclosion to reduce their activity and were tested within 2-7 days of eclosion. Before the experiments, the moths were kept at room temperature $\left(25-28{ }^{\circ} \mathrm{C}\right)$ for at least $10 \mathrm{~min}$. The results of these three types of localization experiments are compared in the next section.

\subsection{Experimental results}

The time-series data of the robot's behavior, the sensor information, and the behavioral data of the silkworm moth acquired using the AIL system, and the sensory stimulus presentation 
state are shown in Fig. 8. Figure 8(a) shows the Euclidean distance from the odor source and the change in the output of the odor sensor, and Fig. 8(b) shows the change in the heading angle, the value of the wind sensor, and the result of wind direction estimation. Figure 8(c) shows the translational and angular velocities of the silkworm moth and the timing of the sensory stimulus presentation. Figure 9 shows the success rate and time required for the three types of localization experiments. We performed Fisher's exact test $(p<0.05)$ to analyze the success rate and the Steel-Dwass test $(p<0.05)$ for the time required for localization. We found no significant difference in the success rate [Fig. 9(a)]. Regarding the required time, there was no significant difference between free walking and the extended AIL system. However, it was found that condition (2) required a significantly longer time than the other conditions [Fig. 9(b)].

The search trajectories and average velocities for the three types of localization experiments are shown in Fig. 10. The successful search trajectory shown is one that was localized in the average search time, while the failed trajectory shown is a typical example. The trajectories under the free-walking and robot-connected conditions show relatively linear motion, whereas

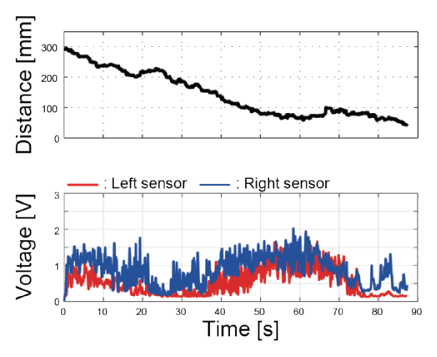

(a)

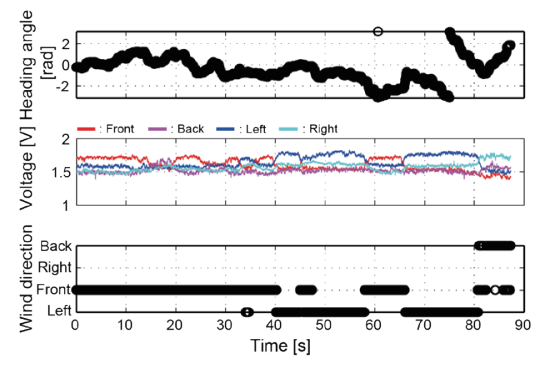

(b)

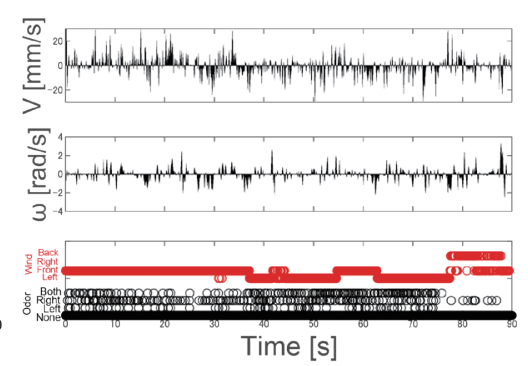

(c)

Fig. 8. (Color online) Results of localization experiments using the extended AIL system. (a, b) Robot data. (a) Euclidean distance from the odor source and the change in response of the odor sensor. (b) Changes in heading angle and value of the wind sensor, and the estimation result of the wind direction. (c) Silkworm moth data showing the translation and angular velocity, and the presentation timing of the odor and wind stimulus.

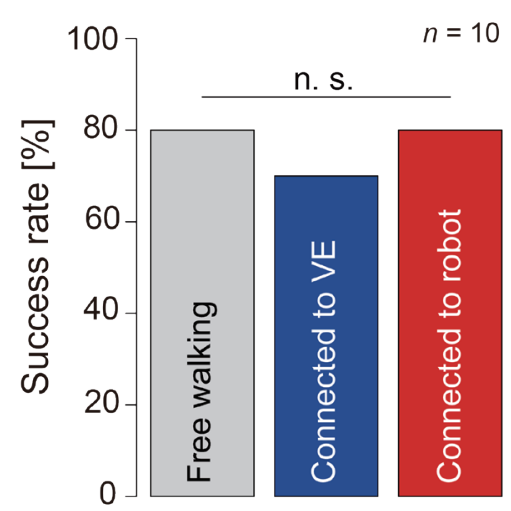

(a)

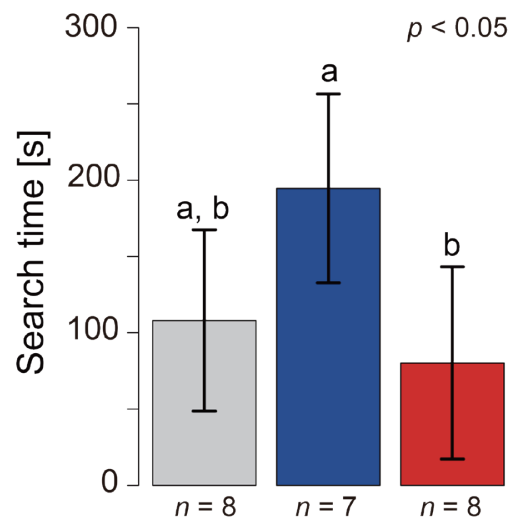

(b)

Fig. 9. (Color online) Success rate and required time of localization experiment. (a) The search success rate did not differ significantly among the experimental systems. (b) The required time for the AIL system connected to the VE was the longest. 


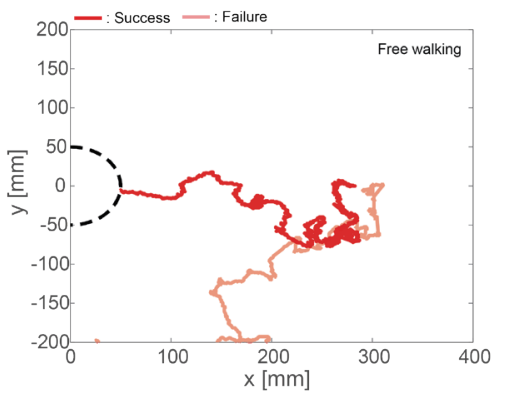

(a)

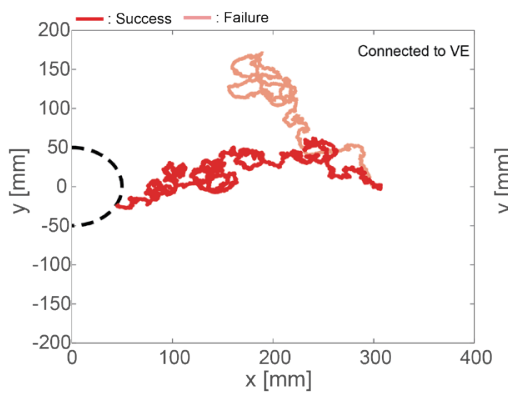

(b)

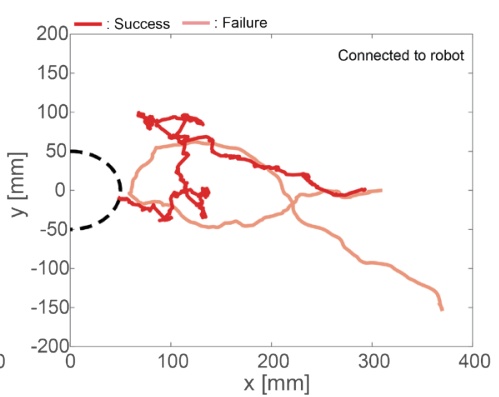

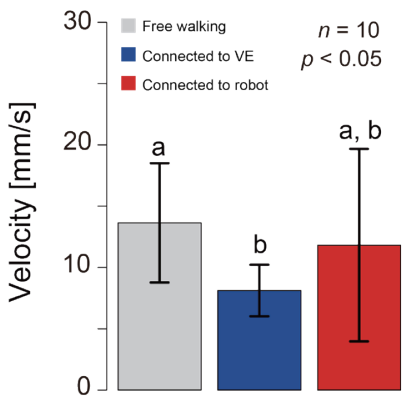

(d)
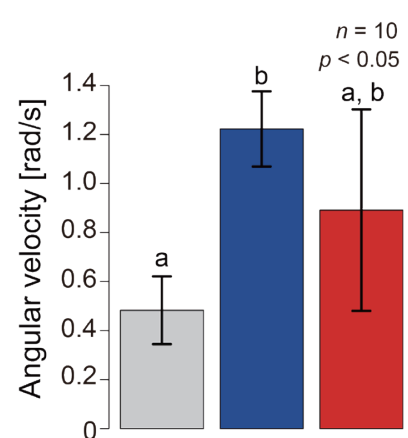

(e) (c)

Fig. 10. (Color online) Comparison of trajectory and movement speed in each localization experiment. (a-c): Trajectories under the free walking, VE-connected, and robot-connected conditions, respectively. (d, e): Comparison of translational velocity and angular velocity, respectively. We performed the Steel-Dwass test $(p<0.05)$ for each velocity data.

those under the VE-connected condition show considerable rotational motion. Figure 10(d) shows that the translational velocity of the VE-connected condition is lower than those of the robot-connected and free-walking conditions. Moreover, the angular velocity of the VEconnected condition is higher than those of the robot-connected and free-walking conditions [Fig. 10(e)]. The mean angular velocity was calculated from the absolute value of the angular velocity, from which we found that under the robot-connected condition, the behavioral characteristics were similar to those in free walking. To quantitatively evaluate the trajectories during localization, we employed density plots of the transit probability (DPTP) ${ }^{(26)}$ to visually represent the localization trajectories. The DPTP is a 2D histogram used to convert the localization field into a grid world and to express how much of an agent has passed through each grid. Figures 11(a)-11(c) respectively show the DPTP for the free-walking experiment, the experiment with a connection to the VE, and the experiment with a connection to the robot, respectively. The yellow color in the DPTP indicates a high probability, the blue color indicates a low probability, and the white area indicates an unreachable area. According to Fig. 11, the localization trajectory of the experiment with the robot connected to the VE did not spread far in the crosswind direction compared with the other conditions. This suggests that there were phenomena such as odor residuals that could not be fully expressed by the odor diffusion model used in the VE. We evaluated the index of similarity between these DPTPs using the earth mover's distance (EMD) ${ }^{(30)}$ tool. EMD is an index that measures the distance between two probability distributions in a statistical manner and is calculated using the Wasserstein metric. 


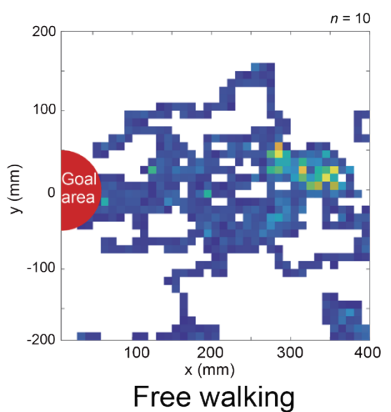

(a)

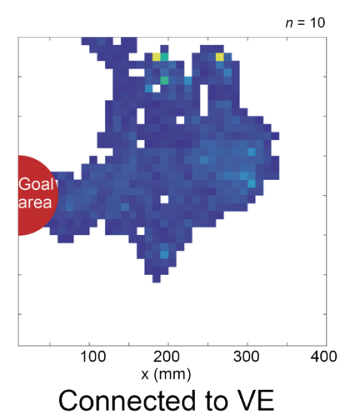

(b)

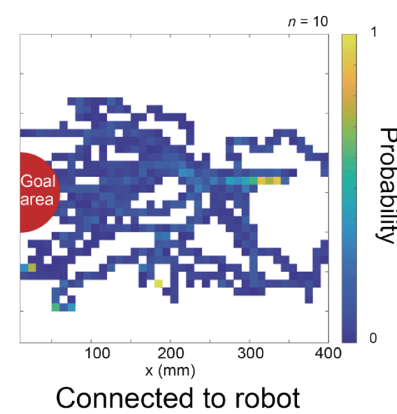

(c)

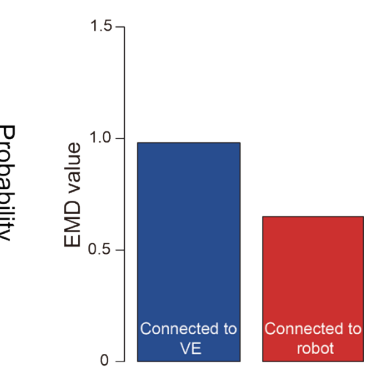

(d)

Fig. 11. (Color online) (a-c) DPTP of localization trajectories. (d) Similarity calculation result for each experimental system. The AIL system connected to the robot has a trajectory resembling that of free walking.

When the two distributions to be compared are $P$ and $Q$, EMD is calculated as

$$
\operatorname{EMD}(P, Q)=\frac{\sum_{i=1}^{m} \sum_{j=1}^{n} f_{i, j}^{*} d_{i, j}}{\sum_{i=1}^{m} \sum_{j=1}^{n} f_{i, j}},
$$

where $\boldsymbol{f}_{\boldsymbol{i}, \boldsymbol{j}}$ is the flow from $\boldsymbol{p}_{\boldsymbol{i}}$ to $\boldsymbol{q}_{\boldsymbol{j}}, \boldsymbol{d}_{\boldsymbol{i}, \boldsymbol{j}}$ is the Euclidean distance, and $\boldsymbol{f}_{\boldsymbol{i}, \boldsymbol{j}}^{*}$ is the optimal flow. The calculations were carried out using OpenCV's EMD function. Because EMD measures the similarity index by calculating the distance between two distributions, the smaller the EMD value, the higher the similarity. We chose EMD for analysis because the DPTP is a twodimensional distribution. We calculated the EMD between (1) free walking and connection to the VE, and (2) free walking and connection to the robot. The results obtained by EMD analyses are shown in Fig. 11(d). These results suggest that the trajectories when connected to the robot are similar to those during free walking, and that the extended AIL system receives the same sensory input as that during free walking and outputs the localization behavior.

Because the direction in which the insect is moving is important for the localization behavior, we obtained the heading angle histogram during the localization behavior (Fig. 12). The radial direction of the heading angle histogram represents the frequency. The $0^{\circ}$ direction of the heading angle histogram indicates the frequency of the upwind direction. Figures 12(b)-12(d) suggest that the frequency of the heading angle in the upwind direction is higher under the experimental conditions of free walking and the connection to the robot than for connection to the VE. In the case of connection to the VE, the frequency is higher for directions other than the windward direction. We performed the Watson-Williams test $(p<0.05)$ on the three heading angle histograms using MATLAB Toolbox for Circular Statistics. ${ }^{(31)}$ No significant difference was found between the heading angle histograms of the free walking and the connected robot; all other combinations showed significant differences. This indicates that free walking and the extended AIL tend to show similarities in terms of both the localization path and heading angle. 


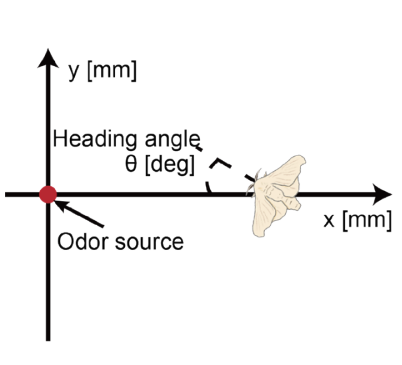

(a)

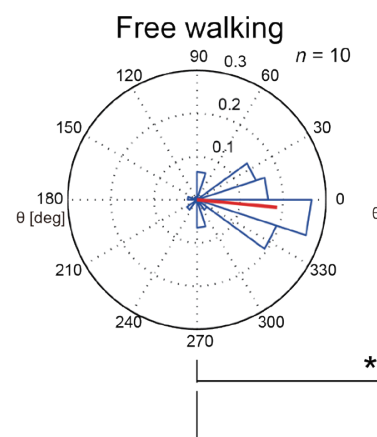

(b)

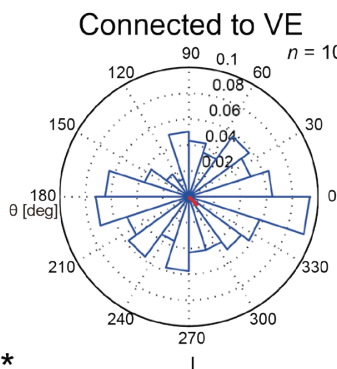

n. S.

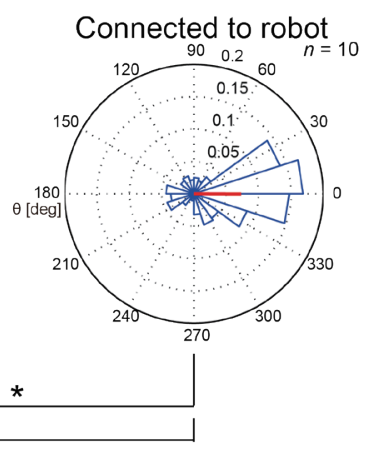

(d)

Fig. 12. (Color online) Heading angle histogram during localization behavior. (a) Definition of heading angle. (b-d) Heading angle histograms for free walking, connected to VE, and connected to robot. We performed the WatsonWilliams test $\left(^{*}: p<0.05\right)$ on these three heading angle histograms.

Next, we show the experimental results when providing a single sensory stimulus (odor) using the extended AIL system. For comparison, we illustrate the results of the AIL system when a combination of multiple sensory information is provided. Figures 13(a)-13(e) respectively show the search success rate, search time, localization trajectory, DPTP during single sensory stimulation, and EMD value for the free-walking experiment. Compared with the case of multiple sensory stimuli, the success rate was lower and the search time was longer in the case of single-odor stimuli [Fig. 13(a) and 13(b)]. According to the localization trajectory, in the case of a single sensory stimulus, there was a tendency to get lost in the middle of the search, and in most cases, the time limit was exceeded [Fig. 13(c)]. By calculating the EMD between the freewalking [Fig. 11(a)] and single-sensory-stimulus [Fig. 13(d)] cases, we found that the similarity was quite low [Fig. 13(e)]. This suggests that the use of multiple sensory stimuli rather than a single sensory stimulus, even when using the AIL, results in greater matching with the freewalking localization behavior.

Hence, by utilizing the extended AIL system, it is possible to elicit insect behaviors that are similar to free walking. It also allows us to measure the relationship among the receiving time, the number of sensory inputs, and the behavior output. By introducing robot and VR technologies into biological experiments, we were able to demonstrate the possibility of measuring the hidden adaptability of an insect in response to environmental dynamics.

\section{Discussion and Conclusion}

In this study, we augmented the conventional AIL system by connecting a multimodal VR device to a ground-running robot to measure the relationship between sensory input and behavioral output. Note that the AIL is effective in quantifying physical phenomena, such as odor or wind flow, which are difficult to describe mathematically. We found more similarities in the localization trajectories obtained by the extended AIL system to the free-walking experiment than to the trajectories obtained using a VR device connected to the VE. The odor field in the 


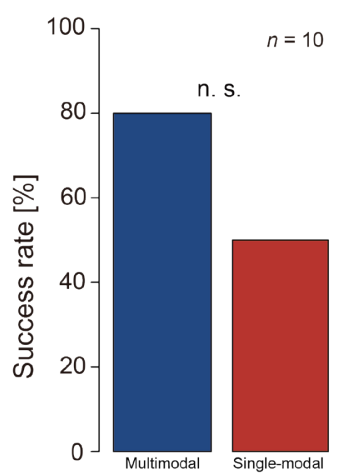

(a)

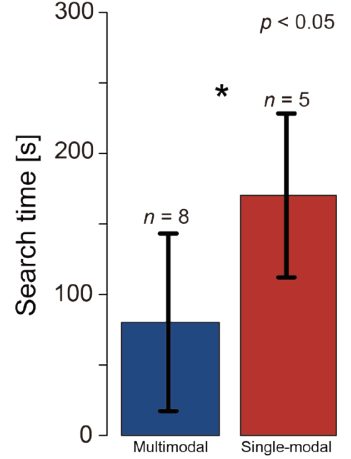

(b)

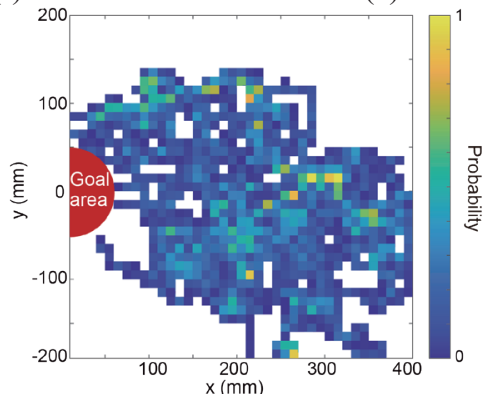

(d)

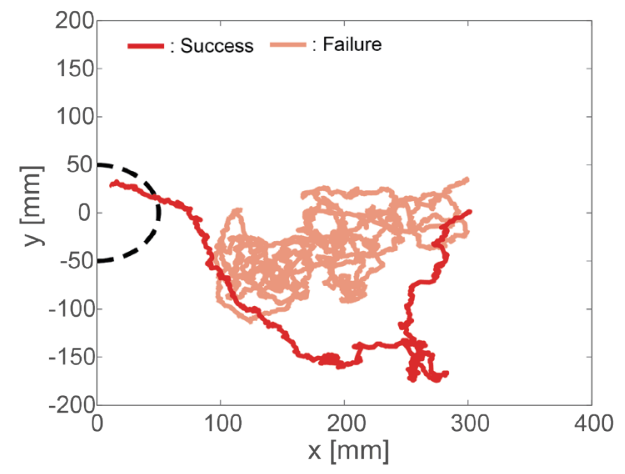

(c)

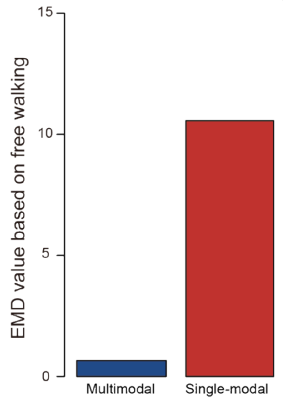

(e)

Fig. 13. (Color online) Comparison of multimodal and single-modal experiments. (a) Success rate. (b) Localization time. (c) Example of single-modal trajectory. (d) Single-modal (odor only) DPTP. (e) Comparison of EMD values based on free-walking experiments.

VE reconstructed the odor diffusion from the odor source based on the PIV measurement method to make it as close to the real world as possible. However, because of the limitation of the camera that captures the fine particles (smoke), it was not possible to observe the behavior of a small amount of fine particles beyond a certain distance from the odor source. Hence, the odor diffusion was not the same as in the real world, which is one reason why the search trajectory of the VE experiment did not resemble that of free walking. However, in the VE, the environment itself can be manipulated by the experimenter, and because it is a useful tool for measuring adaptive behavior when the environment is modified, we claim that the VE method is also an effective tool. Thus, it is important to choose an appropriate method depending on the research purpose. According to the results of the comparative experiments in this study, the AIL system (1) can reconstruct the real environment via each sensory stimulator and (2) can present realistic environmental information that is difficult to represent mathematically to an insect via connection to a robot that moves in the real environment. In the future, we will attempt to simultaneously measure internal states (calcium imaging/neural activity) and localization behavior because the multimodal VR device is based on the conventional tethered behavior measurement system.

Previous studies have used an insect-mounted measurement system, ${ }^{(26,32)}$ which gives mobility to the tethered behavioral measurement system. The advantage of this system is that it allows the intentional modification of the behavioral output of an insect to investigate its 
robustness and adaptability. It thus highlights the effect of sensory feedback on behavior by intervening in the motor system of the insect. However, using the insect-mounted method makes it difficult to intervene in the sensory system of the insect. Hence, it was not possible to investigate behavioral changes in response to manipulation of the sensory inputs or the delay time of the system. The advantage of the extended AIL system is that it facilitates manipulation of both the motor and sensory systems of the insect, thus expanding the range of possible investigations compared with the insect-mounted approach. The AIL method allows modulation of the scale of the robot, the spatial arrangement of the sensors on the robot, and the delay time for each of the sensory and motor systems. Therefore, the AIL system is a promising approach for understanding the system structure that supports the adaptive behavior of insects by studying the effects of specific manipulations in the motor and sensory systems.

Because this study focused on the extended AIL system, a few research limitations remain, such as in inputting the wind speed and odor. The environmental wind speed and odor concentration were not fed back to the silkworm moth as sensory stimuli. The system needs to be improved to include differences in wind speed and concentration because these may induce behavioral changes in the insect. Moreover, the odor input is easily affected by the scale of the agent, and the search field as its dispersal is significantly influenced by the airflow. The shape of the robot also affects the degree of diffusion when the odor collides with the robot. In this study, we were not able to determine whether the results obtained are general or dependent on a specific shape or scale size. We plan to investigate these issues using tools such as computational fluid dynamics and PIV in the future.

\section{Acknowledgments}

This work was partially supported by JKA through its promotion funds from AUTORACE and JSPS KAKENHI under grant nos. JP19K14943 and JP19H02104.

\section{References}

1 Y. Yamada, K. Ito, T. Tsuji, K. Otani, R. Kobayashi, Y. Watanabe, and S. Hiryu: Adv. Rob. 33 (2018) 3. https:// doi.org/10.1080/01691864.2019.1573470

2 K. L. Baker, M. Dickinson, T. M. Findley, D. H. Gire, M. Louis, M. P. Suver, J. V. Verhagen, K. I. Nagel, and M. C. Smear: J. Neurosci. 38 (2018) 44. https://doi.org/10.1523/JNEUROSCI.1668-18.2018

3 B. Webb and A. Wystrach: Current Opinion Insect Sci. 15 (2016). https://doi.org/10.1016/j.cois.2016.02.011

4 F. L. Moël and A. Wystrach: Current Opinion Insect Sci. 42 (2020). https://doi.org/10.1016/j.cois.2020.10.006

5 S. J. Huston and V. Jayaraman: Curr. Opin. Neurobiol. 21 (2011)4. https://doi.org/10.1016/j.conb.2011.05.030

6 D. J. Harvey, T. F. Lu, and M. A. Keller: IEEE Trans. Rob. 24 (2008)2. https://doi.org/10.1109/TRO.2007.912090

7 P. K. Kaushik, M. Renz, and S. B. Olsson: PNAS 117 (2020) 22. https://doi.org/10.1073/pnas.1912124117

8 H. Naik, R. Bastien, N. Navab, and I. D. Couzin: IEEE Trans. Visual Comput. Graphics 26 (2020) 5. https://doi. org/10.1109/TVCG.2020.2973063

9 M. Yamada, H. Ohashi, K. Hosoda, D. Kurabayashi, and S. Shigaki: eLife. 10 (2021). https://doi.org/10.7554/ eLife.72001

10 S. Shigaki, M. R. Fikri, C. Hernandez Reyes, T. Sakurai, N. Ando, D. Kurabayashi, R. Kanzaki, and H. Sezutsu: Adv. Rob. 32 (2018) 17. https://doi.org/10.1080/01691864.2018.1511473

11 J. S. Carrel: Science 175 (1972) 4027. https://science.sciencemag.org/content/175/4027/1279

12 A. G. Witney and B. Hedwig: J. Exp. Biol. 214 (2011) 1. https://doi.org/10.1242/jeb.044800

13 N. Nagaya, N. Mizumoto, M. S. Abe, S. Dobata, R. Sato, and R. Fujisawa: PloS One 12 (2017) 6. https://doi. org/10.1371/journal.pone. 0177480 
14 S. Shigaki, S. Fukushima, D. Kurabayashi, T. Sakurai, and R. Kanzaki: Bioinspiration Biomimetics 12 (2016) 1. https://doi.org/10.1088/1748-3190/12/1/016005

15 P. Pansopha, N. Ando, and R. Kanzaki, R: J. Exp. Biol. 217 (2014) 10. https://doi.org/10.1242/jeb.090266

16 S. Shigaki, S. Haigo, C. H. Reyes, T. Sakurai, R. Kanzaki, D. Kurabayashi, and H. Sezutsu: Bioinspiration Biomimetics 14 (2019) 4. https://doi.org/10.1088/1748-3190/ab1d34

17 K. Okajima, S. Shigaki, T. Suko, D. N. Luong, C. H. Reyes, Y. Hattori, K. Sanada, and D. Kurabayashi: J. R. Soc. Interface 18 (2021) 181. https://doi.org/10.6084/m9.figshare.c.5545140

18 E. González, F. Marzal, A. Minãna, and M. Doval: Environ. Prog. 27 (2008) 3. https://doi.org/10.1002/ep.10287

19 S. Shigaki, M. R. Fikri and D. Kurabayashi: Sensors 18 (2018) 11. https://doi.org/10.3390/s18113720

20 T. Fujiwara, T. Kazawa, T. Sakurai, R. Fukushima, K. Uchino, T. Yamagata, S. Namiki, S. S. Haupt, and R. Kanzaki: J. Neurosci. 34 (2014) 50. https://doi.org/10.1523/JNEUROSCI.2319-14.2014

21 S. Shigaki, K. Okajima, K. Sanada, and D. Kurabayashi: IEEE Rob. Autom. Lett. 4 (2019) 3. https://doi. org/10.1109/LRA.2019.2921948

22 C. Loudon and M. A. Koehl: J. Exp. Biol. 203 (2000) 19. https://doi.org/10.1242/jeb.203.19.2977

23 T. Yoshihara, S. Shigaki, and D. Kurabayashi: Trans. JSME 83 (2017) 853 (in Japanese). https://doi.org/10.1299/ transjsme.17-00108

24 T. Lochmatter, X. Raemy, L. Matthey, S. Indra, and A. Martinoli: 2008 IEEE Int. Conf. Robotics and Automation (2008). https://doi.org/10.1109/ROBOT.2008.4543357

25 D. Harvey, T. F. Lu, and M. Keller: Australasian Conf. Robotics and Automation (2003). https://www.araa.asn. au/acra/acra2003/papers/16.pdf

26 N. Ando, S. Emoto, and R. Kanzaki: Bioinspiration Biomimetics 8 (2013) 1. https://doi.org/10.1088/17483182/8/1/016008

27 G. A. Kane, G. Lopes, J. L. Saunders, A. Mathis, and M. W. Mathis: eLife 9 (2020). https://doi.org/10.7554/ eLife.61909

28 R. Yanagawa, S. Shigaki, and D. Kurabayashi: Proc. SICE Annu. Conf. (2008).

29 R. J. Adrian: Experiments in Fuids 39 (2005) 2. https://doi.org/10.1007/s00348-005-0991-7

30 Y. Rubner, C. Tomasi, and L. J. Guibas: Int. J. Comput. Vision 40 (2000) 2. https://doi. org/10.1023/A:1026543900054

31 P. Berens: J. Stat. Software 31 (2009) 10. https://doi.org/10.18637/jss.v031.i10

32 J. Wessnitzer, A. Asthenidis, G. Petrou, and B. Webb: Conf. Autonomous Robotic Systems (2011). https://doi. org/10.1007/978-3-642-23232-9 1 